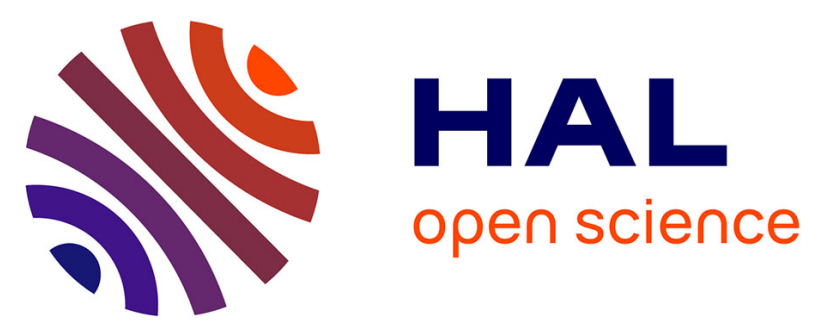

\title{
AIS-based Evaluation of Target Detectors and SAR Sensors Characteristics for Maritime Surveillance
}

\author{
Ramona-Maria Pelich, Nicolas Longépé, Grégoire Mercier, Guillaume
}

Hajduch, René Garello

\section{- To cite this version:}

Ramona-Maria Pelich, Nicolas Longépé, Grégoire Mercier, Guillaume Hajduch, René Garello. AISbased Evaluation of Target Detectors and SAR Sensors Characteristics for Maritime Surveillance. IEEE Journal of Selected Topics in Applied Earth Observations and Remote Sensing, 2015, 8 (8), pp.3892 - 3901. 10.1109/JSTARS.2014.2319195 . hal-01258834

\section{HAL Id: hal-01258834 \\ https://hal.science/hal-01258834}

Submitted on 19 Jan 2016

HAL is a multi-disciplinary open access archive for the deposit and dissemination of scientific research documents, whether they are published or not. The documents may come from teaching and research institutions in France or abroad, or from public or private research centers.
L'archive ouverte pluridisciplinaire HAL, est destinée au dépôt et à la diffusion de documents scientifiques de niveau recherche, publiés ou non, émanant des établissements d'enseignement et de recherche français ou étrangers, des laboratoires publics ou privés. 


\title{
AIS-based Evaluation of Target Detectors and SAR Sensors Characteristics for Maritime Surveillance
}

\author{
Pelich Ramona, Student Member, IEEE, Longépé Nicolas, Grégoire Mercier, Senior Member, IEEE, Hajduch \\ Guillaume, and René Garello, Fellow, IEEE
}

\begin{abstract}
This paper studies the performances of different ship detectors based on adaptive threshold algorithms. The detection algorithms are based on various clutter distributions and assessed automatically with a systematic methodology. Evaluation using large datasets of medium resolution SAR images and AIS (Automatic Identification System) data as ground truths allows to evaluate the efficiency of each detector. Depending on the datasets used for testing, the detection algorithms offer different advantages and disadvantages. The systematic method used in discriminating real detected targets and false alarms in order to determine the detection rate, allows us to perform an appropriate and consistent comparison of the detectors. The impact of SAR sensors characteristics (incidence angle, polarization, frequency and spatial resolution) is fully assessed, the vessels' length being also considered. Experiments are conducted on Radarsat- 2 and CosmoSkymed ScanSAR datasets and AIS data acquired by coastal stations.
\end{abstract}

\section{Index Terms}

Synthetic Aperture Radar (SAR), ship detection, Constant False Alarm Rate (CFAR), statistic distribution, non-parametric, Automatic Identification System (AIS).

\section{INTRODUCTION}

$\mathbf{S}$ YNTHETIC Aperture Radar (SAR) imagery offers nowadays a great potential in observing and monitoring the maritime environment. Knowledge about vessels position and type may serve for a wide range of applications, such as maritime traffic safety, fisheries control and border surveillance.

Although the literature and the applications [1] developed in this area are vast, the evolution of SAR sensors capabilities and the availability of data produces new research issues. Ship detection on SAR images has been intensively studied using data acquired from the Canadian Radarsat-1 satellite and the European satellites: ERS-1/2 (European Remote Sensing) and ENVISAT (Environmental Satellite). The detection capabilities from the aforementioned C-band data have been studied in [2], [3], [4]. More recent spaceborne SAR sensors, namely the Canadian Radarsat-2 (RS-2) satellite, the Italian CosmoSkyMed (CSK) satellites constellation or the German TerraSAR-X satellite, may provide additional capabilities for ship detection. The diversity of polarizations, new emitting X-band frequency along with the capability of Left/Right looking with higher spatial resolutions may provide additional assets for maritime surveillance.

For operational services, ships detection systems usually involve the following steps [1], [3], [5]: land masking, detection algorithm, classification and human supervision. For land masking a dedicated coast line database is used with a buffer zone of variable size depending on operational services. The detection phase consists in using an adaptive threshold algorithm within a sliding window while the classification step is of high importance to reduce the false alarm rate. Human supervision aims to assess and discriminate the detection results in view of the final detection report. This study focuses on the detection algorithms, no post-processing being applied here.

The aim of this paper is to compare and analyze the results of different detection algorithms applied to SAR intensity images. To the best of our knowledge this is the first time that different detection algorithms based on various clutter distributions are assessed automatically with a systematic and consistent methodology. First we use the classical adaptive threshold algorithm - the Constant False Alarm Rate (CFAR) detector based on the Gaussian or the Gamma distribution. For multi-look intensity images, the clutter distribution is known to be Gamma-distributed, converging to Gaussian distribution for large number of independent looks. We propose a third detection algorithm, which arises from a non-parametric approach [6], that allows to detect a signal with unknown distribution in the presence of white Gaussian noise.

The proposed algorithms are evaluated on different datasets obtained from Radarsat-2 and CosmoSkyMed (Scan SAR mode). To evaluate the detection's performances we use coastal Automatic Identification System (AIS) data as ground truth. However, the range coverage of AIS receiver is limited by Very High Frequency (VHF) propagation loss. For instance, messages from vessels at a distance of 20 Nautical Miles to the AIS stations, will be hardly received. Therefore, we propose a methodology to consider the propagation loss for the automatic assessment of ship detection reports.

R. Pelich, N. Longépé and G. Hajduch are with Collecte Localisation Satellites (CLS) - Avenue La Pérouse - 29280 Plouzané - France, (e-mail: \{rpelich, nlongepe, ghajduch \}@cls.fr).

R. Pelich, G. Mercier and R. Garello are with Institut Mines Telecom - Telecom Bretagne, CNRS UMR 6285 LabSTICC, Technopôle Brest-Iroise - CS 83818 - 29238 Brest Cedex 3 - France (e-mail:\{rm.pelich, gregoire.mercier, rene.garello \}@ telecom-bretagne.eu). 
The remainder of this paper is organized as follows. Section II describes the proposed detection algorithms and the new methodology to compare SAR detection reports with AIS data in view of the reliable assessment of ship detectors. Section III presents the experimental results and the analysis of ship detection performances. Finally, Section IV gives the conclusions.

\section{Detection Algorithms, SAR And AIS Data}

\section{A. Detections Methodologies}

The following briefly describes the detection algorithms. The CFAR detector is very often used in ship detection and many papers have already treated the subject (ref. [7] and [1] give a very good synthesis of existing papers). We remind that designing an adaptive threshold algorithm (CFAR in our case) implies to compare pixels from the target cell within a sliding window (pixels under test $x_{\text {test }}$ ) to a threshold depending on the statistics of the surrounding area (background cell $x_{B}$ ). A guard cell area is used for ensuring that no pixel of an extended target is included in the background cell. In order to compute the threshold, statistics in the background cell and the corresponding probability density function (PDF) for a desired probability of false alarm (PFA), see Eq. (1), should be estimated.

$$
\text { PFA }=\int_{\tau}^{\infty} f\left(x / x_{B}\right) \mathrm{d} x .
$$

Two classical methods for estimating parameters of statistical distributions are the Method of Moments (MoM) and the Maximum Likelihood Estimation (MLE) [8]. In this study, the MoM has been used and the corresponding statistical parameters of the background area are the mean, $\mu_{B}$, and the standard deviation, $\sigma_{B}$.

a) Gaussian CFAR detection: if we consider as statistical model the Gaussian distribution, the CFAR detector becomes $x_{\text {test }} \gtrless \mu_{B}+\sigma_{B} \tau$, and finding $\tau$ involves only the PFA as a parameter for solving Equation (1).

b) Gamma CFAR detection: in the case of the Gamma distribution, the detector's principle is given by

$x_{\text {test }} \gtrless \mu_{B} \tau$, with $\tau$ retrieved by [9] and [10] using relation (2). $L$ is the order parameter of the gamma distribution and the effective number of looks used for multi-look processing. We can use for $L$ 's value, either the number of looks given in the sensors documentation, or its estimation from the background statistics (equivalent number of looks (ENL) defined by: $\left(\frac{\mu_{B}}{\sigma_{B}}\right)^{2}$.

$$
\mathrm{PFA}=\frac{\Gamma(L, \tau)}{\Gamma(L)}
$$

c) Non-parametric detection: The non-parametric detector [6] aims at detecting a signal of unknown distribution in presence of white Gaussian noise, using a simple approach that requires only the estimation of the noise variance. We consider the following hypothesis :

$\left\{\begin{array}{l}H_{0}: Y \sim N\left(0, \sigma^{2}\right) \\ H_{1}: Y=S+X, \lambda_{u} \leq|S|, X \sim N\left(0, \sigma^{2}\right)\end{array}\right.$

In our approach $S$ represents the target information, a signal of unknown distribution and $X$ is the background - white Gaussian noise. $X$ and $S$ are considered independent. Assuming that $S$ is of very small dimensions comparing to $X$ (size $N$ ), according to theory, the maximum of $X$ has a strong probability of being close to $\lambda_{u} \cdot \lambda_{u}=\sigma \sqrt{2 \ln N}$, the so-called universal threshold [11], [12]. We assume that the probability of occurrence of $H_{1}$ is less than a certain $p$ - the probability of presence of signal in noise $(\leq 0.5)$. Under all these assumptions a non-parametric threshold, $\lambda_{n}$, representing a lower-bound for $|S|$, is defined as [6]: $\lambda_{n}=\sigma \xi\left(\frac{\lambda_{u}}{\sigma}, p\right)$, with:

$$
\xi(a, p)=\frac{a}{2}+\frac{1}{a}\left[\ln \frac{1-p}{p}+\ln \left(1+\sqrt{1-\frac{p^{2}}{(1-p)^{2}} e^{-a^{2}}}\right)\right] .
$$

Finding $\lambda_{u}$ or $\lambda_{n}$ implies the estimation of $\sigma$, which is the standard deviation of the background, in our case. Since we use intensity images, the assumption $X \sim N\left(0, \sigma^{2}\right)$ is not valid. But multi-looked SAR intensity images are the sum of $L$ terms $\left(I_{L}^{2}+Q_{L}^{2}\right)$, where $I_{L}$ and $Q_{L}$ represent the real and imaginary components of radar scatter for each look. Because $I_{L}$ and $Q_{L}$ are independently Gaussian distributed, we can easily find a relationship between $\sigma_{I / Q}$ and $\sigma_{\text {intensity }}$ (the last one can be estimated) and employ it for finding $\lambda_{u}$ or $\lambda_{n}$.

d) Fusion of detectors: As each detector considers parametric and non-parametric models, their fusion may be considered. Several combinations based on T-norm and T-conorm fuzzy operators were tested. Nevertheless, their benefits remain limited, so that they will not be further documented in this paper.

\section{B. Integration of AIS data flows for ship detection assessment}

To assess the ship detection performance with a comprehensive dataset, a systematic methodology should be proposed to automatically integrate AIS data flows as a reliable ground truth. To this end, we have modeled [13] the reception capabilities 


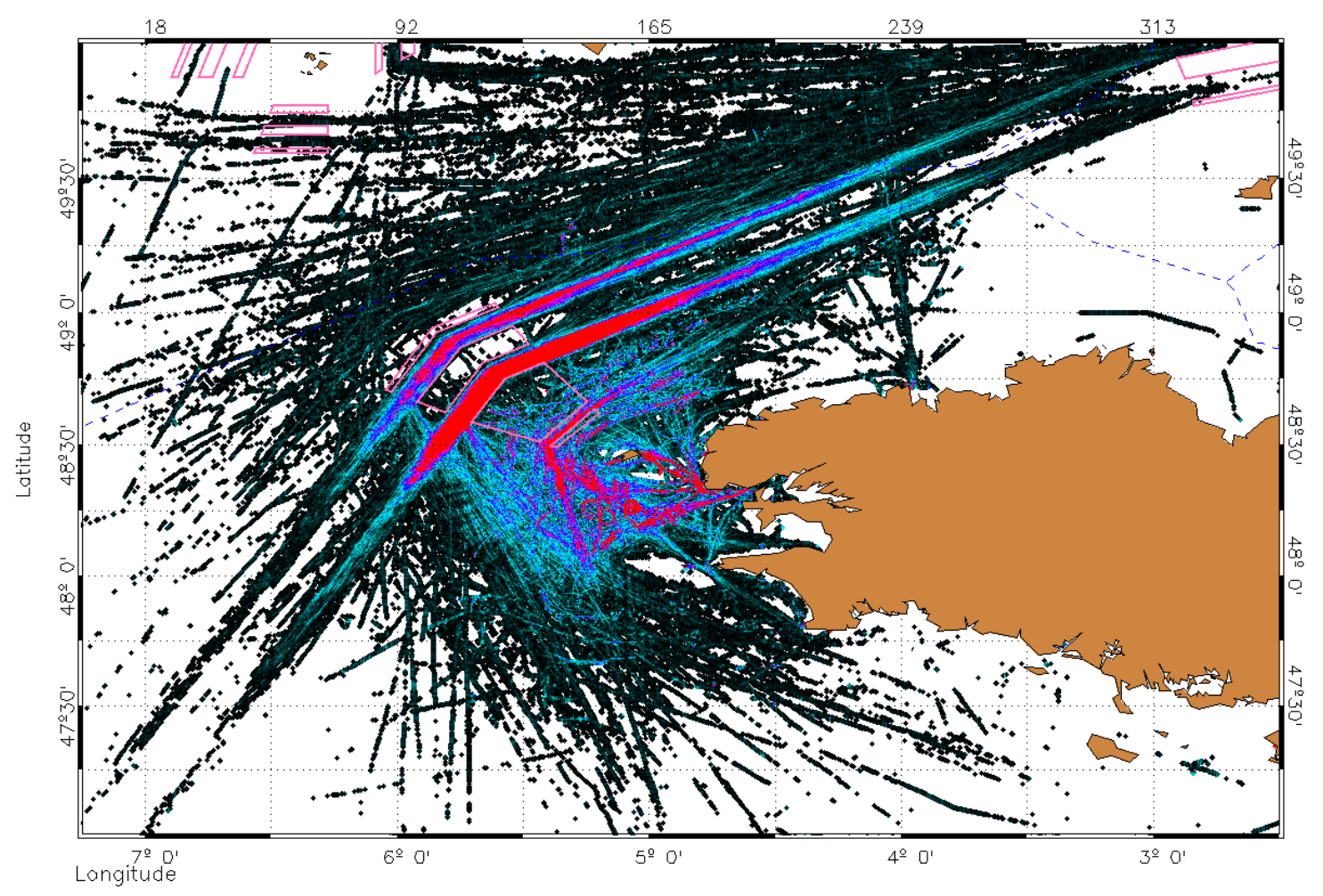

Number of decaded AIS positions

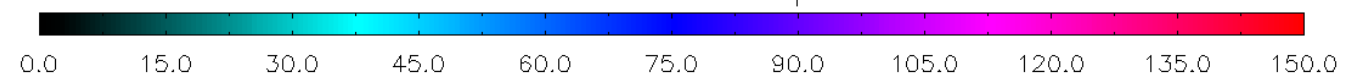

Figure 1: Accumulation of AIS messages received by the Enez Eusa AIS station during July 2011 (cell resolution for counting vessels $\left.-0^{\circ} 0.25^{\prime}\right)$.

of coastal AIS receivers located in the British Channel and nearby the Ushant island. For each AIS station, an accumulation of all the AIS messages received during a long period (e.g. one month) is first extracted. The impact of the propagation range loss can be observed in Figure 1. Assuming a constant flow of vessels in the Traffic Separating Scheme (TSS) between the ascending and descending routes, and also within the scheme itself, the number of received positions per range distance can be computed and the probability of receiving AIS messages is then fitted by an affine function $\psi$, depending on the distance from the AIS receivers. Considering the modeling of AIS stations, SAR ship detection reports are compared to AIS data in order to evaluate the detection algorithms. The comparison is performed in the AIS area determined by the modeling of AIS stations. Only SAR targets whose correspondent $\psi$ is above 0 are used to assess the detector's performances. Figure 2 gives the modeled confidence indicator of receiving AIS messages.

Vessels positions given by SAR reports and AIS data are used to perform the comparison. For each SAR image, the corresponding AIS data is first extracted with a time range of $\pm 3 \mathrm{~h}$ with respect to the SAR image acquisition time. For each vessel AIS data flow is interpolated at the time of SAR acquisition, using either a bi-linear interpolation when 2 or more positions are available ( $\pm x$ minutes), or the route/speed when one position is available. For a better coupling, AIS points are correctly positioned on the SAR image taking into account the exact acquisition time in the azimuth direction and the possible azimuthal offset related to their radial speed. Simple iterative process based on distance criteria is then applied. A minimal distance between the AIS and SAR locations has to be attained in order to consider a match. Targets not attaining the condition are considered as false alarms if SAR targets, or missed targets if AIS targets.

All detected SAR targets with a corresponding AIS position are considered as valid SAR detections. Otherwise, detected SAR targets without a corresponding AIS position are classified as false alarms. Finally, AIS positions without matching detected SAR targets are considered as missed detections.

This classification allows us to estimate the effective detection probability, $\mathrm{P}_{\mathrm{d} \text {-eff }}^{\mathrm{AIS}}$, and the effective probability of false alarm, 


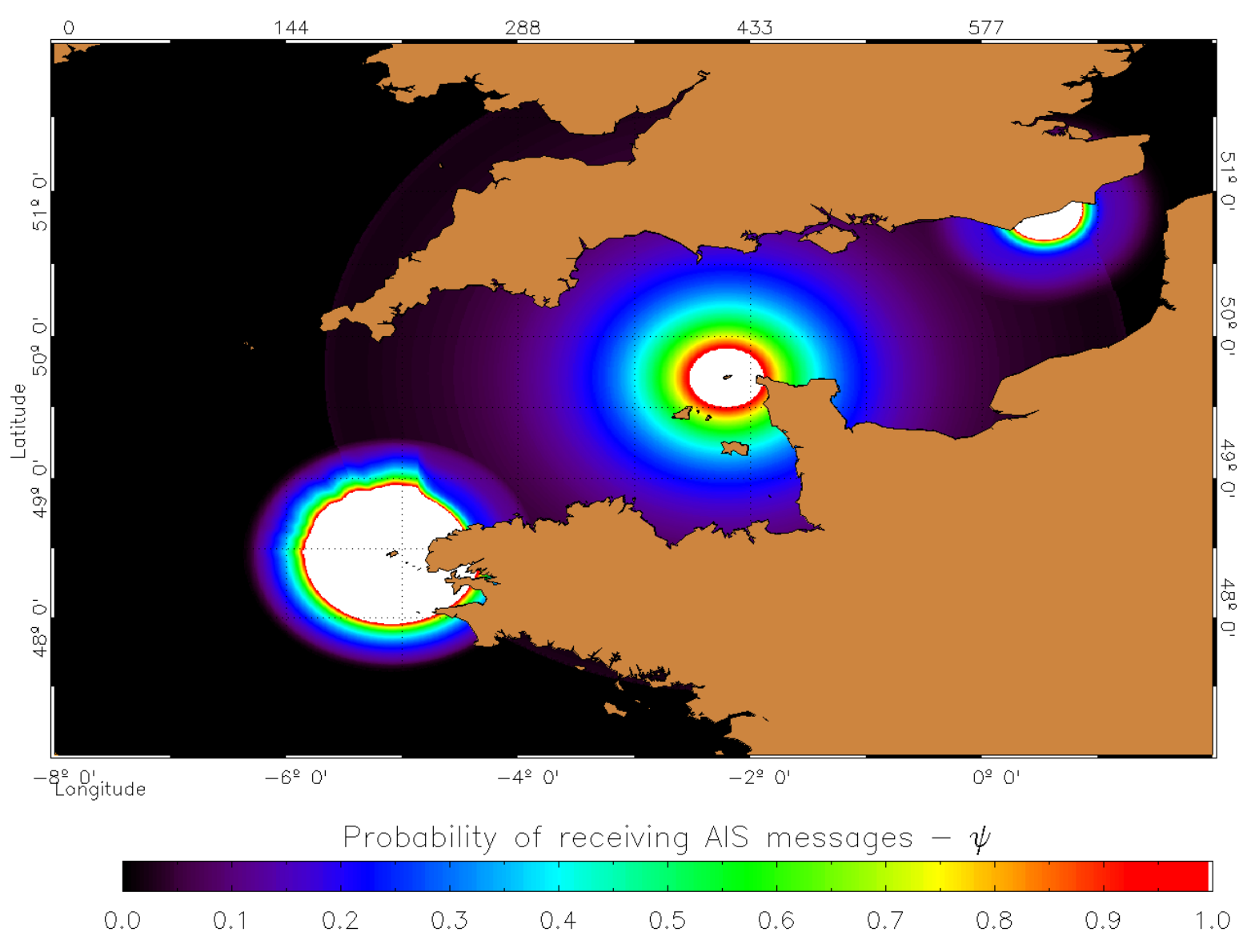

Figure 2: Area covered by AIS stations.

PFA $_{\text {eff }}^{\mathrm{AIS}}$. In this context $\mathrm{P}_{\mathrm{d} \text {-eff }}^{\mathrm{AIS}}$ is estimated as follows:

$$
\mathrm{P}_{\mathrm{d}-\mathrm{eff}}^{\mathrm{AIS}}=\frac{\sum_{i=1}^{N_{m}} \psi_{i}}{\sum_{j=1}^{N_{\mathrm{AIS}}} \psi_{j}}
$$

where $N_{m}$ is the total number of detected SAR targets matched with AIS data and $N_{\text {AIS }}$ is the number of all existent AIS targets. $\psi$ represents the probability of receiving AIS messages in their corresponding targets locations (see Figure 2).

The effective value of $\mathrm{P}_{\mathrm{d} \text {-eff }}^{\text {AIS }}$ is estimated by the following ratio:

$$
\mathrm{PFA}_{\mathrm{eff}}^{\mathrm{AIS}}=\frac{\left(\sum_{i=1}^{N_{\mathrm{FA}}} \psi_{i}\right) \times N_{\mathrm{PPT}}}{\sum_{k=1}^{N_{\mathrm{PA}}} \psi_{k}-\left(\sum_{j=1}^{N_{\mathrm{AIS}}} \psi_{j}\right) \times N_{\mathrm{PPT}}}
$$

where $N_{\mathrm{FA}}$ is the total number of SAR targets classified as false alarms. $N_{\mathrm{PA}}$ represents the number of pixels in the area covered by the AIS stations, delimited by the land masking process. $N_{\text {PPT }}$ is defined as the maximum number of pixels per target with respect to image resolution and represents the area of a circle having the diameter equal to the length of the longest desired target. This definition of $N_{\text {PPT }}$ is used in order to be able to compare the PFA $\mathrm{eff}_{\mathrm{ef}}$ for the various detectors. $N_{\mathrm{PPT}}$ cannot be defined as the number of pixels detected by the SAR based detector for each particular target due to the fact that for the CFAR detector the threshold comparison is cell based while for the non-parametric detector the threshold comparison is pixel based and thus the number of pixels per target will not be comparable between detectors.

AIS carriage regulations require sending AIS messages for all ships exceeding 300 tons engaged on international voyages, cargo ships exceeding 500 tons not engaged on international voyages and all passenger ships, whatever their size. Since the AIS dataset may not contain all existent ships in the area, the estimations of $\mathrm{P}_{\mathrm{d} \text {-eff }}^{\mathrm{AIS}}$ and PFA $\mathrm{eff}^{\mathrm{AIS}}$ are influenced by this. For instance, a real ship detected on the SAR image, but with no correspondent AIS position, will be classified as false alarms, causing a decrease of $\mathrm{P}_{\mathrm{d} \text {-eff }}^{\mathrm{AIS}}$ and increase of $\mathrm{PFA}_{\mathrm{eff}}^{\mathrm{AIS}}$. Given the large number of targets used in our study this kind of cases are 
Table I: SAR Datasets characteristics

\begin{tabular}{||c||c|c|c|c|}
\hline Dataset (number of images) & Resolution $(\mathbf{r n g} \times \mathbf{a z})[\mathbf{m}]$ & Polarization & Number of looks & Frequency band \\
\hline Radarsat-2 ScanSAR Wide (200) & $72.1-160 \times 100$ & $\mathrm{VV}$ & 8 & $\mathrm{C}$ \\
Radarsat-2 ScanSAR Narrow (40) & $37.7-79.9 \times 60$ & $\mathrm{HH}$ & 4 & $\mathrm{C}$ \\
CosmoSkymed ScanSAR Huge Region (35) & $100 \times 100$ & $\mathrm{VV}$ & 15 & $\mathrm{X}$ \\
\hline
\end{tabular}

minor and will slightly change detection rates.

\section{EXPERIMENTAL RESULTS}

To evaluate the detection algorithms we used three different datasets of SAR intensity images. Table I provides a summary of sensors characteristics corresponding to each dataset [14], [15].

\section{A. General parameters settings}

For the CFAR sliding window, the size of the different cells (target, buffer and background) is chosen depending on the image resolution and the minimal/maximal sizes of targets under consideration. In our experiments, the buffer area is related to the dimensions of the biggest desired target (for instance $400 \mathrm{~m}$ - world longest ship in service), and thus is defined by a circle with a proportional radius. The square-shaped background area is then constrained by this dimension, enlarged by a factor of $\sqrt{2}$. As for the non-parametric detector, estimating the variance of the background uses the same circle-box architecture with same size, for the sake of a coherent assessment between methods.

Concerning the number of looks $(L)$, as a parameter for the Gamma CFAR and the non-parametric detectors, several possibilities were tested to set its value: the one given in the sensors documentation, the ENL estimated globally for each image or locally at pixel level. For the Gamma detector, the use of the ENL estimated locally gave the best detection performances. Background statistics of the image $\left(\mu_{B}\right.$ and $\left.\sigma_{B}\right)$ are estimated using the sliding CFAR window in order to remain consistent with the detection scheme. However, for the non-parametric detector, the $L$ value given in sensors documentations was used, giving better results.

\section{B. Detector's performances, first level analysis}

In order to analyze the overall performances of the different detection algorithms we use the Receiver Operating Characteristic (ROC) curves. We remind that the parameter which controls the CFAR detectors is the desired PFA and for the non-parametric detector, is $p$ (the probability of presence of signal in noise). By giving different values for these parameters we plot the ROC curves and analyze the detectors behavior. For the desired PFA we give values from $10^{-2}$ to $10^{-16}$, adding that for lower values the number of detections remains constant. For $p$, values between $10^{-5}$ and 0.5 are given, the lower the value of $p$, the non-parametric threshold $\lambda_{n}$ tends to the universal threshold $\lambda_{u}$.

Figure 3 shows the results obtained by the detection algorithms applied on our three datasets. Depending on the prior PFA and $p$, the estimated $\mathrm{PFA}_{\text {eff }}^{\mathrm{AIS}}$ and $\mathrm{P}_{\mathrm{d} \text {-eff }}^{\mathrm{AIS}}$ range from $10^{-4}$ to $10^{-2}$, and from $40 \%$ to $80 \%$, respectively.

We notice that the Gaussian and Gamma CFAR detectors give similar results, but one given PFA value would result in different $\left(\mathrm{PFA}_{\text {eff }}^{\mathrm{AIS}}, \mathrm{P}_{\mathrm{d} \text {-eff }}^{\mathrm{AIS}}\right)$ values with the Gaussian or the Gamma assumptions. The results over the RS-2 ScansSAR Narrow dataset show that for PFA equal to $10^{-5}$ the Gaussian detector gives $\left(\mathrm{PFA}_{\mathrm{eff}}^{\mathrm{AIS}}=10^{-3.1}, \mathrm{P}_{\mathrm{d} \text {-eff }}^{\text {AIS }}=0.68\right)$ while the Gamma detector gives $\left(\mathrm{PFA}_{\mathrm{eff}}^{\mathrm{AIS}}=10^{-3.4}, \mathrm{P}_{\mathrm{d} \text {-eff }}^{\mathrm{AIS}}=0.6\right)$.

We can observe that the Non-parametric detector gives slightly better performances than the CFAR detectors for CSK and RS-2 ScanSAR Wide but lower performances for RS-2 ScanSAR Narrow.

Regarding the polarization type of our datasets, CSK and RS-2 ScanSAR Wide contain both VV polarization data, while RS-2 ScanSAR Narrow contains HH polarization data. According to [1], [16], HH polarization is better for ship detection than VV polarization due to a higher ship-sea contrast. The detection results obtained with the RS-2 ScanSAR Narrow dataset are significantly better than with the RS-2 ScanSAR Wide dataset $\left(\mathrm{P}_{\mathrm{d} \text {-eff }}^{\mathrm{AIS}} \in[0.5,0.8]\right.$ vs $\left.\mathrm{P}_{\mathrm{d} \text {-eff }}^{\text {AIS }} \in[0.4,0.65]\right)$. Beside the higher resolution of the RS-2 ScanSAR Narrow dataset, the HH polarization plays an important role for the ship detection.

If we compare the performances by image type, we notice that the best results are achieved on CSK data, and the lower ones for RS-2 ScanSAR Wide, even though both have almost the same resolution and same VV polarization. For CSK, X-band could be a potential benefit compared to C-band for ship detection: sea surface backscattering at X-and C-bands are in the same order for standard wind speed $(7 \mathrm{~m} / \mathrm{s}$ ), but backscattering from targets could be higher at X-band (even if relatively badly known). We may expect to get the best results from the dataset having the best SAR characteristics known for ship detectability (higher resolution, HH polarization,...), but the first level analysis with ROC curves may lead to contradictory conclusion with global results better for the CSK Huge Region data than RS-2 ScanAR Narrow data. An in-depth analysis of the datasets characteristics explaining the obtained results is given in the subsequent sections. The differences may be caused by the higher number of looks for Huge Region CSK (15) compared to ScanSAR RS-2 (8 for Wide, 4 for Narrow). Out of the 


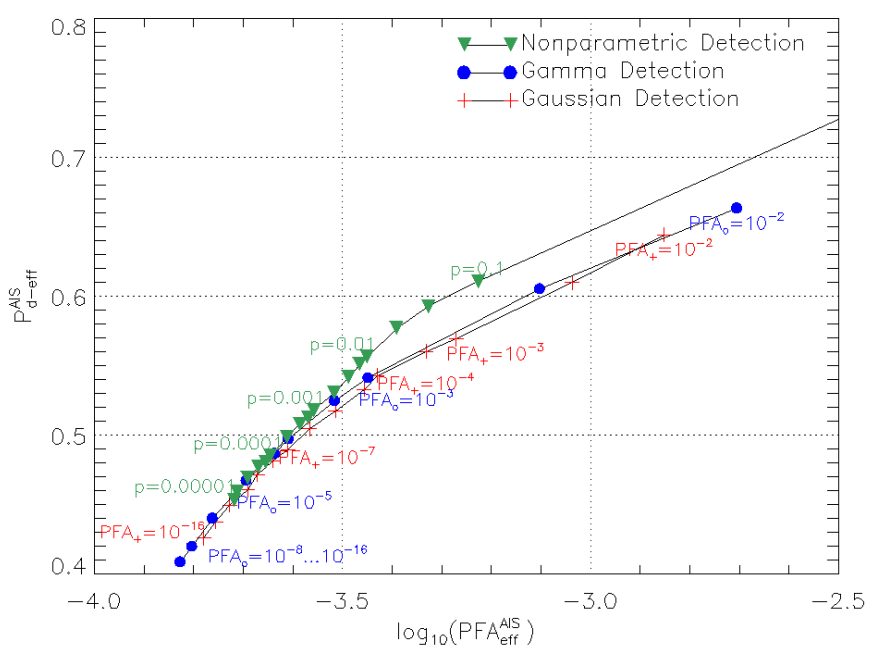

(a) 200 RS-2 ScanSAR Wide images

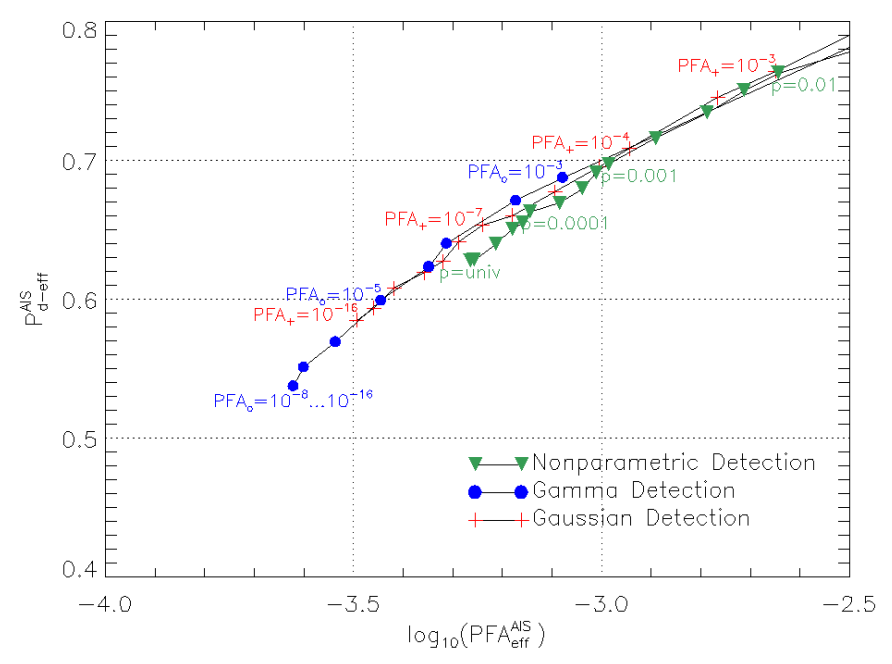

(b) 40 RS-2 ScanSAR Narrow images

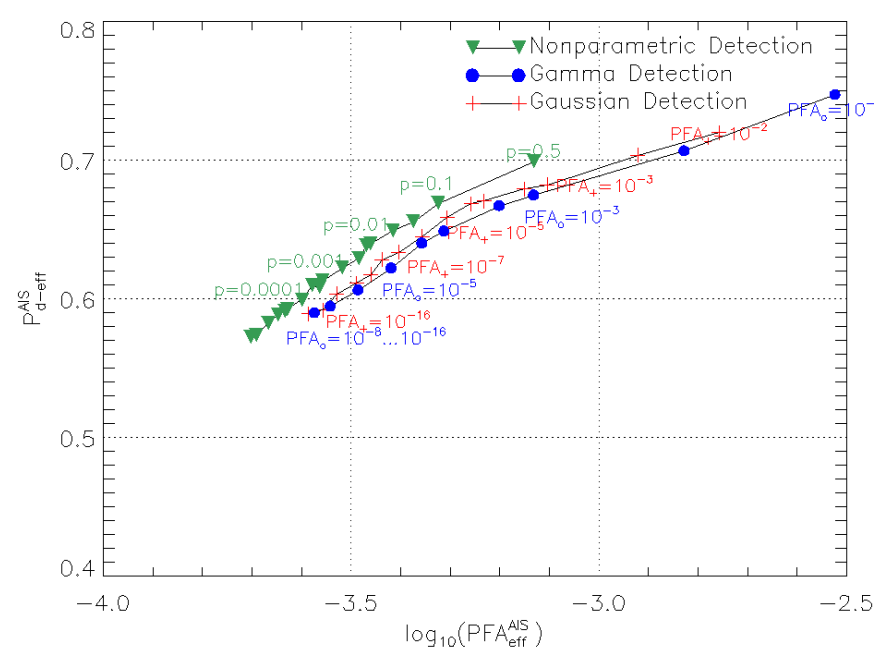

(c) 35 CSK ScanSAR Huge Region images

Figure 3: Receiver Operating Characteristic - ROC curves, comparison of detector's performances by algorithm.

sensors/modes characteristics, the corresponding ranges of incidence angle, weather conditions (e.g. wind speed) and vessels length over our datasets may impact the detection performances.

\section{Influence of different key parameters on the detection rate}

Our datasets contain a large amount of SAR images. This allows us to estimate the probability of detection depending on different key parameters, such as SAR imaging characteristics or meteorological conditions. The influence of the incidence angle, wind speed (weather condition) and vessel length, which are key parameters for ship detection, has been assessed in this study. At this stage, it should be mentioned that we do not perform a multi-parameter analysis but instead a one-by-one assessment of the detection rate. The first approach would have required a much larger dataset. Knowing that for similar $\left(\mathrm{PFA}_{\text {eff }}^{\text {AIS }}, \mathrm{P}_{\mathrm{d} \text {-eff }}^{\text {AIS }}\right)$ values the detected SAR targets are almost the same no matter the employed detector, the analysis of the influence of different key parameters on the detection rate will be realized using results of the Gaussian detector.

1) Incidence angle: The incidence angle is a relevant parameter for ship detection algorithms, knowing that low incidence angles are favorable to ocean backscattering, while larger values reduce it and increase the ship Radar Cross Section (RCS) [16], [17]. The ship detection performances over our datasets will necessarily be correlated with the distribution of the incidence angles (given in figure 4).

Regarding the incidence angle range of the RS-2 ScanSAR Narrow dataset we observe that almost $50 \%$ are lower than $30^{\circ}$ which rends a substantial part of SAR data less appropriate for the detection. Instead, the ScanSAR Wide images show a wider range of the incidence angle than ScanSAR Narrow images and a considerable part (70\%) of the corresponding incidence angles are greater than $30^{\circ}$. In terms of incidence angle we state that a high percentage of the RS-2 ScanSAR Wide dataset 


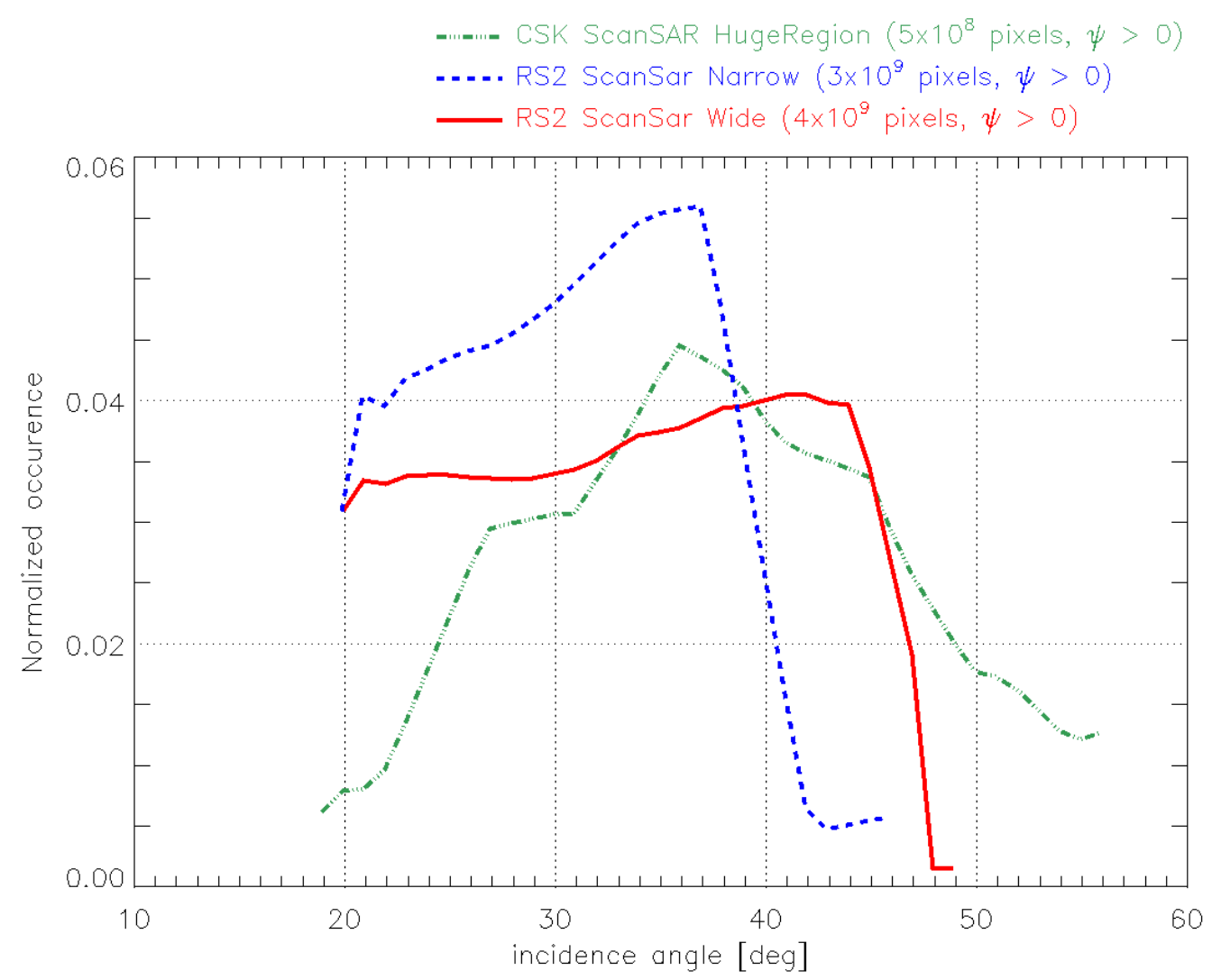

Figure 4: Incidence angles distribution for all the pixels of our dataset with $\psi>0$ (Histogram binsize $=1$ degree, Histogram smooth with mean filter of size 5).

is adequate for ship detection. We notice that for the CSK dataset, a small amount of data has low corresponding incidence angles ( $20 \%$ of data with incidence angles between $20^{\circ}$ and $30^{\circ}$ ) and the range for higher values extends comparing to the RS-2 datasets (up to $50^{\circ}$ ). This makes the CSK dataset better suited for the detection with respect to the incidence angle.

Figure 5 gives the detection performances depending on the incidence angles values. In order to estimate $\mathrm{P}_{\mathrm{d} \text {-eff }}^{\text {AS }}$ and PFA $\mathrm{eff}$ (given in Eqs. (4) and (5)) as a function of the incidence angle, $N_{m}, N_{\mathrm{FA}}, N_{\mathrm{PA}}$ and $N_{\mathrm{AIS}}$ were determined taking into account incremental steps of the incidence angle.

We can observe that $\mathrm{P}_{\mathrm{d} \text {-eff }}^{\mathrm{AIS}}$ increases with the incidence angle, over all datasets, which is in accordance with the fact that ships signature are enhanced at higher incidence angles. The same assumption is confirmed by the low number of detections at low incidence angles, $\mathrm{P}_{\mathrm{d} \text {-eff }}^{\text {IIS }}$ increasing with the incidence angle (for instance for RS-2 ScanSAR Wide and CSK datasets the $\mathrm{P}_{\mathrm{d} \text {-eff }}^{\text {AIS }}$ varies from 0.2 to 0.4 for incidence angles from $20^{\circ}$ to $30^{\circ}$ ).

For $70 \%$ of the RS-2 ScanSAR Wide dataset with corresponding incidence angles greater than $30^{\circ}$, $\mathrm{P}_{\mathrm{d} \text {-eff }}^{\mathrm{AIS}}$ values vary from 0.4 to 0.8 (sub-figure 5- $(a)$ ) while for the rest of data are lower than 0.4 . The reduced detection rate for higher incidence angles may be caused by the other key parameters with impact on detectors performances. For $50 \%$ of the RS-2 ScanSAR Narrow dataset with corresponding incidence angles lower than $30^{\circ}, \mathrm{P}_{\mathrm{d} \text {-eff }}^{\mathrm{AIS}}$ values vary from 0.2 to 0.7 (sub-figure 5 - $(b)$ ) while for the rest of data, the values are between 0.8 and 0.9 . Therefore we can state that for RS-2 ScanSAR Narrow dataset the ship detection is best suited for incidence angles greater than $30^{\circ}$. In case of the CSK images for incidence angles between $30^{\circ}$ to $40^{\circ} \mathrm{P}_{\mathrm{d} \text {-eff }}^{\text {AIS }}$ values vary from 0.5 to 0.8 (sub-figure 5- $(c)$ ) while for higher incidence angles $\left(40^{\circ}\right)$, $\mathrm{P}_{\mathrm{d} \text {-eff }}^{\text {AIS }}$ remains constant around 0.8 achieving the best performances for this dataset.

Intrinsically, the ScanSAR Narrow dataset our only dataset with HH-polarization, which is known to have the lowest sensitivity to sea-clutter and thus better suited for ship detection) provides the best detection capabilities whatever the incidence angle. In the previous part, the global detection rates over this dataset were clearly impacted by the incidence angle distribution, being diminished by the large percentage of SAR data at low incidence angles.

As shown in Figure 5 the values of $\mathrm{PFA}_{\mathrm{eff}}^{\mathrm{AIS}}$ increase with the incidence angle. The corresponding Signal-to-Noise Ratio (SNR) depends upon the incidence angle, as large incidence angles imply a higher likelihood of low SNR [18].

2) Sea surface roughness via wind field information: As the wind blows over the sea surface, it generates capillary-gravity ocean surface waves. These waves increase the surface roughness with respect to wind speed and direction and respond immediately to the ocean surface wind. In the microwave domain, the backscattered signal from the ocean surface directly depends upon the size of these waves. At a given time, the roughness observed on a SAR image is thus directly related to the 


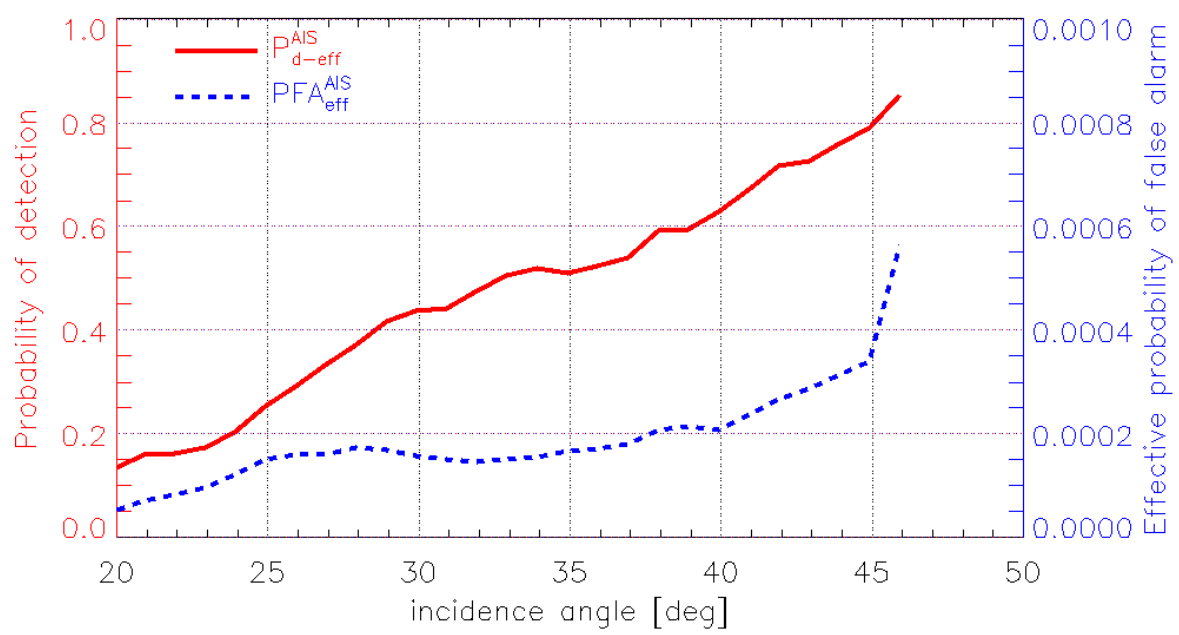

(a) 200 RS-2 ScanSAR Wide images

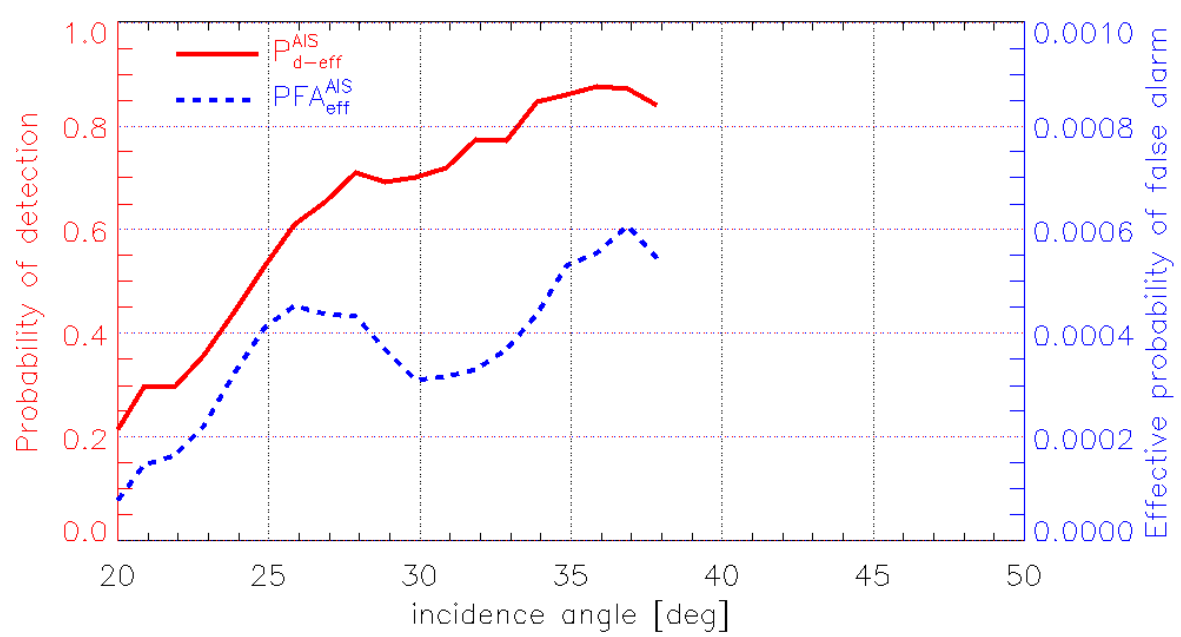

(b) 40 RS-2 ScanSAR Narrow images (for incidence angles $>40^{\circ}$, results are omitted due to the small amount of existent data).

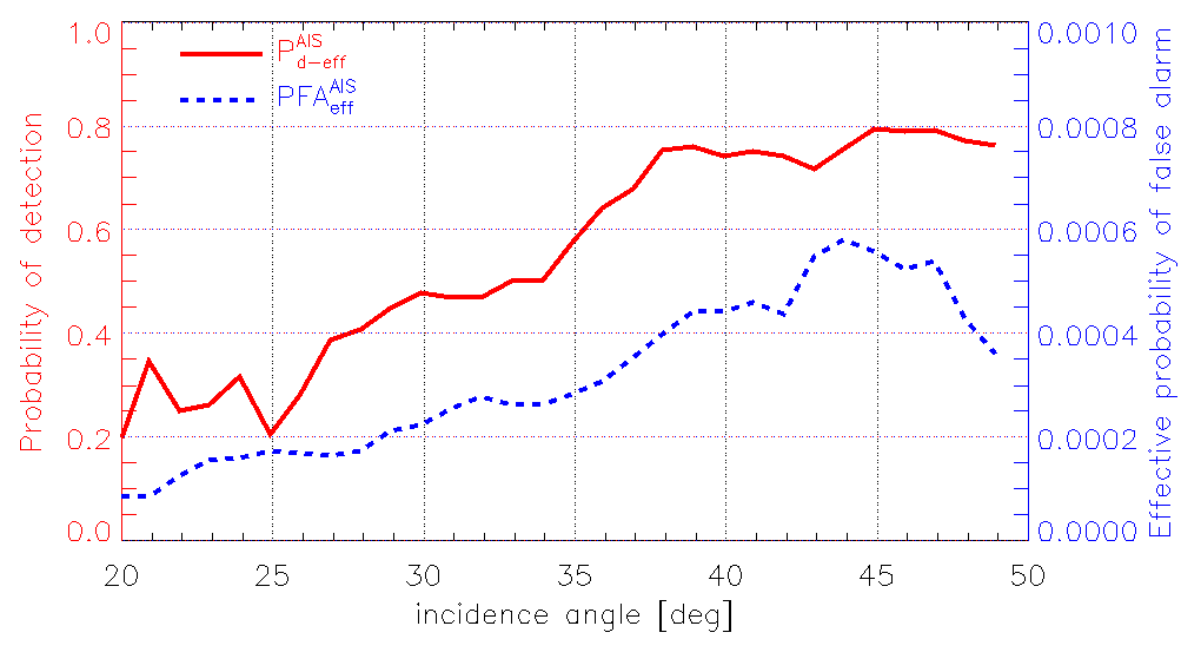

(c) 35 CSK ScanSAR Huge Region images

Figure 5: Detection rate depending on the incidence angle, Gaussian detector $\left(P F A=10^{-7}\right)$.

local and instantaneous wind [18]. Therefore the wind speed being correlated with the sea surface roughness will impact the detection capabilities, the increase of wind speed values implying a decrease of the detection rate [19]. The wind direction and the stability of the atmospheric surface layer are also contributing to the surface roughness. Adding this parameters for 


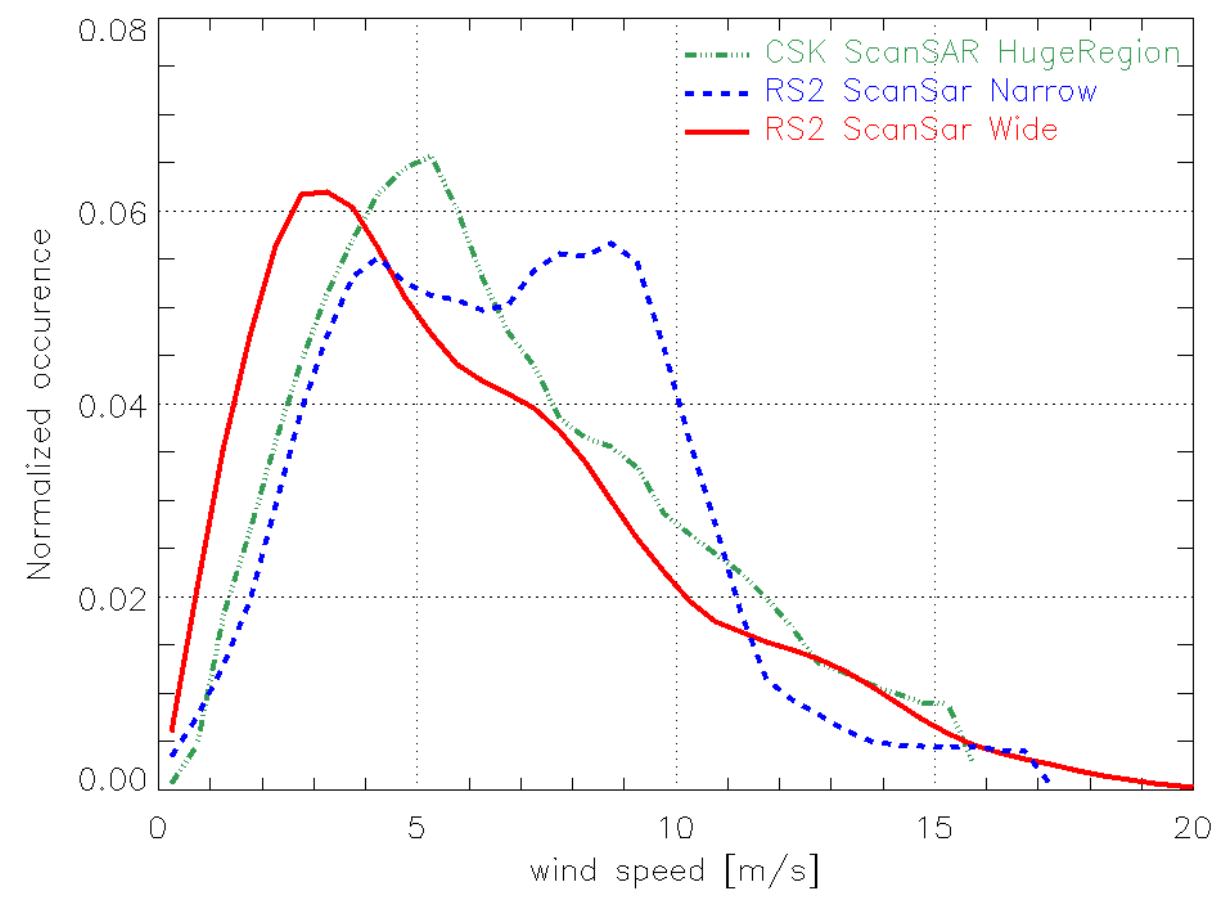

Figure 6: Wind speed distribution for all the pixels of our dataset with $\psi>0$ (Histogram binsize $=1 \mathrm{~m} / \mathrm{s}$, Histogram smooth with mean filter of size 4).

detector's analysis would have required a much larger dataset and increase the complexity for results analysis. Also the potential information extracted from ancillary data demands an elaborate work in order to be integrated automatically in our assessment. In this study we will only analyze the wind speed impact on detection performances using the wind field information provided by National Centers for Environmental Prediction (NCEP) with 3-hour temporal and $0.2^{\circ}$ spatial resolution. All this ancillary information was co-located and interpolated to the SAR image grid, taking the closest temporal available data. Figure 6 gives the wind speed distribution over our datasets for the corresponding SAR images.

The RS-2 ScanSAR Wide dataset contains for almost $50 \%$ of the SAR data, corresponding wind speeds lower than $6 \mathrm{~m} / \mathrm{s}$, which are favorable for the detection, $\mathrm{P}_{\mathrm{d} \text {-eff }}^{\mathrm{AIS}}$ being constant around 0.6 as shown in Figure 7 (the best global detection rate obtained on this dataset - see Figure 3- $(a)$ ). For the other half of the dataset detection rates decrease. This confirm us that for RS-2 ScanSAR Wide the detection rate varies with the wind speed, decreasing for wind speeds values from $6 \mathrm{~m} / \mathrm{s}$ to $20 \mathrm{~m} / \mathrm{s}$. For the RS-2 ScanSAR Narrow dataset we observe that for low wind speed values, $\mathrm{P}_{\mathrm{d} \text {-eff }}^{\text {AIS }}$ varies between 0.7 and 0.8 , which are close to the best global performances over this dataset. As we expected for higher incidence values, $\mathrm{P}_{\mathrm{d} \text {-eff }}^{\text {AIS }}$ decreases. The wide range around $8-10 \mathrm{~m} / \mathrm{s}$ corresponding to a decrease for $\mathrm{P}_{\mathrm{d} \text {-eff }}^{\text {AIS }}$ makes the dataset less favorable for ship detection. Concerning the wind speed values corresponding to CSK dataset, the majority of data is distributed around $5 \mathrm{~m} / \mathrm{s}$ and detection reports

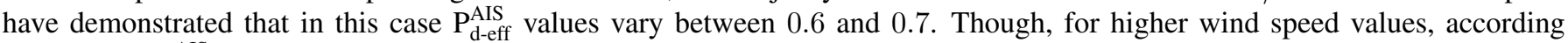
to Figure $7, \mathrm{P}_{\mathrm{d} \text {-eff }}^{\mathrm{AIS}}$ is increasing contradicting the hypothesis of lower detection performances at higher wind speed values. The small amount of data for corresponding high wind speed values could explain it, further analysis of this contradiction being implied in our future work.

As shown in Figure 7, the values of PFA $\mathrm{eff}$ decrease with the wind speed. For low wind speed values, SAR scenes are affected by the sensor's noise, which explains the high number of false alarms.

3) SAR Resolution AND Vessel Length: Spatial resolution of SAR images plays a very important role in evaluating the detection algorithms. In view of Section III-B the detection rate depends strongly on ships size existing in our datasets. Certain ships with a prominent signature can be detected in SAR images and others not, depending on their size with respect to images spatial resolution. In medium resolution SAR imagery, ships signatures do not contain a large amount of pixels, especially for medium and small vessels. The retrieval of ship length from medium resolution SAR imagery, is hardly feasible and may result in erroneous estimates. The AIS data flows provide for the majority of vessels information about their size. The histograms shown in Figure 8 give the distribution of the number of ships, depending on their length (given by AIS information), over our datasets.

For the RS-2 ScanSAR Wide images, with resolution of $72.1-160 \times 100 \mathrm{~m}$, we expect that ships of length lower than $100 \mathrm{~m}$ can be difficultly, even impossibly detected. Figure 9 gives the detection rate depending on ships length, showing that for low ship lengths the detection probability is low. For ship lengths under $50 \mathrm{~m}$, $\mathrm{P}_{\mathrm{d} \text {-eff }}^{\text {AIS }}$ is almost 0 , which is consistent with the resolution of the dataset. We also notice that for ship lengths between $50 \mathrm{~m}$ to $150 \mathrm{~m}$, $\mathrm{P}_{\mathrm{d} \text {-eff }}^{\text {AIS }}$ grows from 0.1 to 0.7 , and 


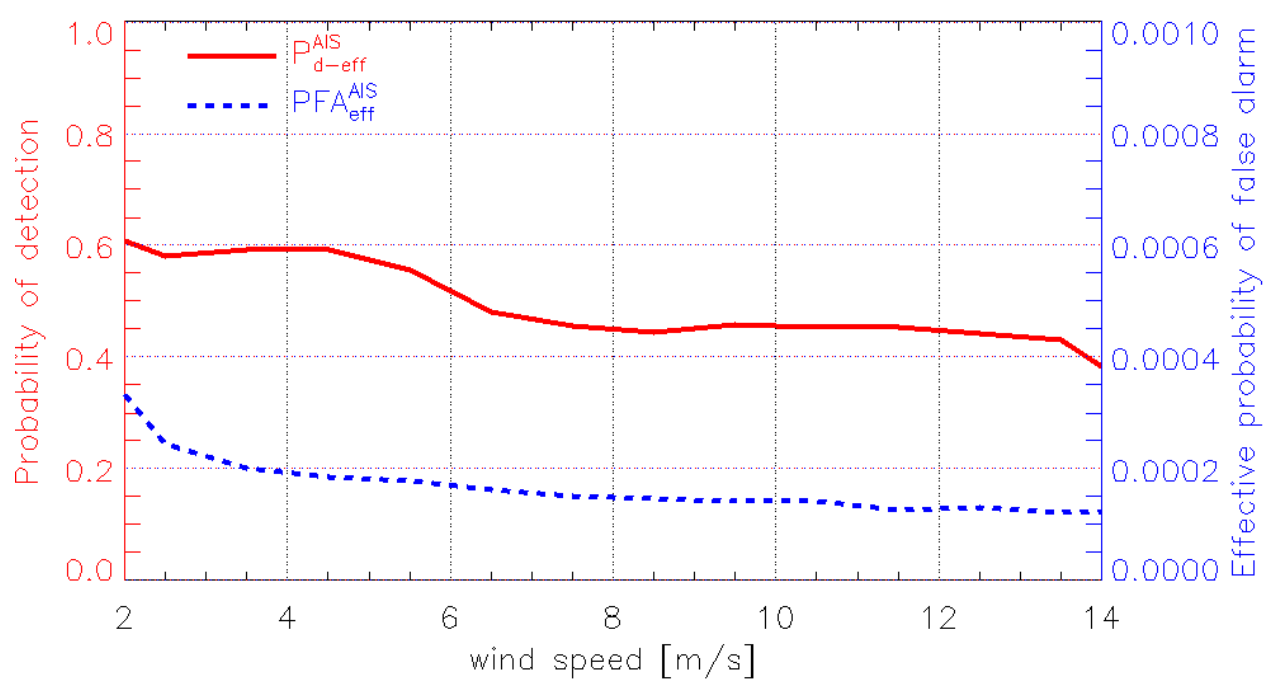

(a) 200 RS-2 ScanSAR Wide images

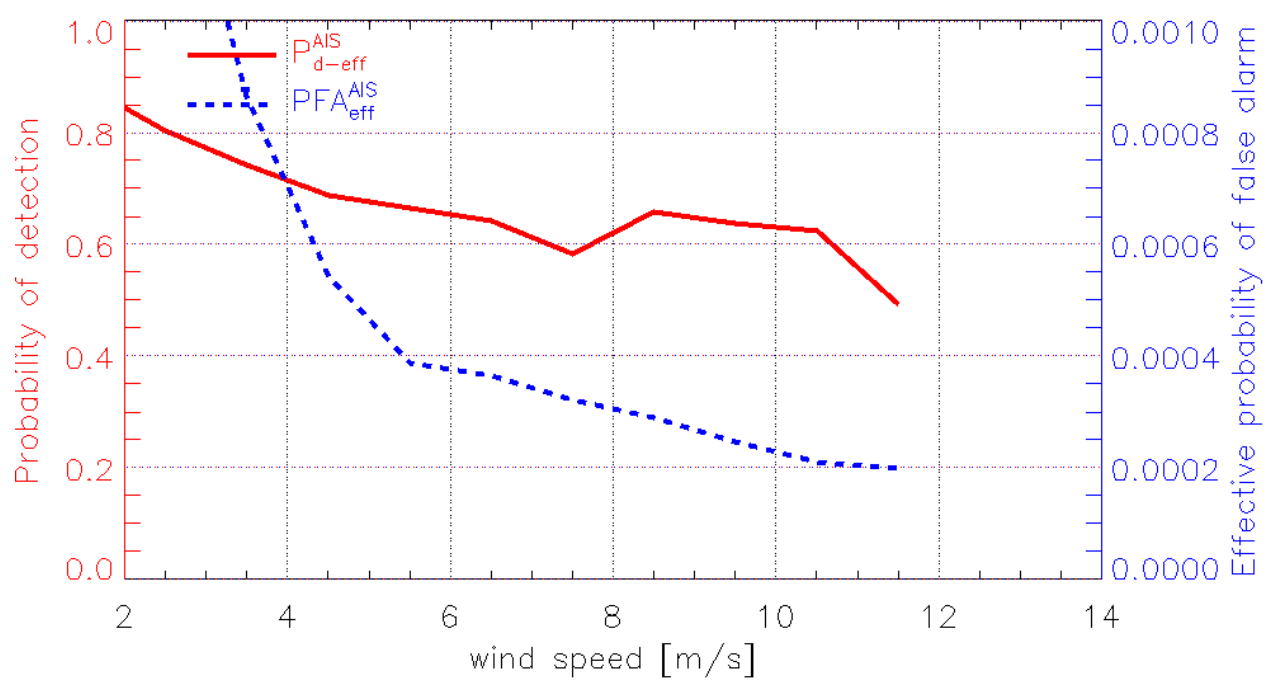

(b) 40 RS-2 ScanSAR Narrow images

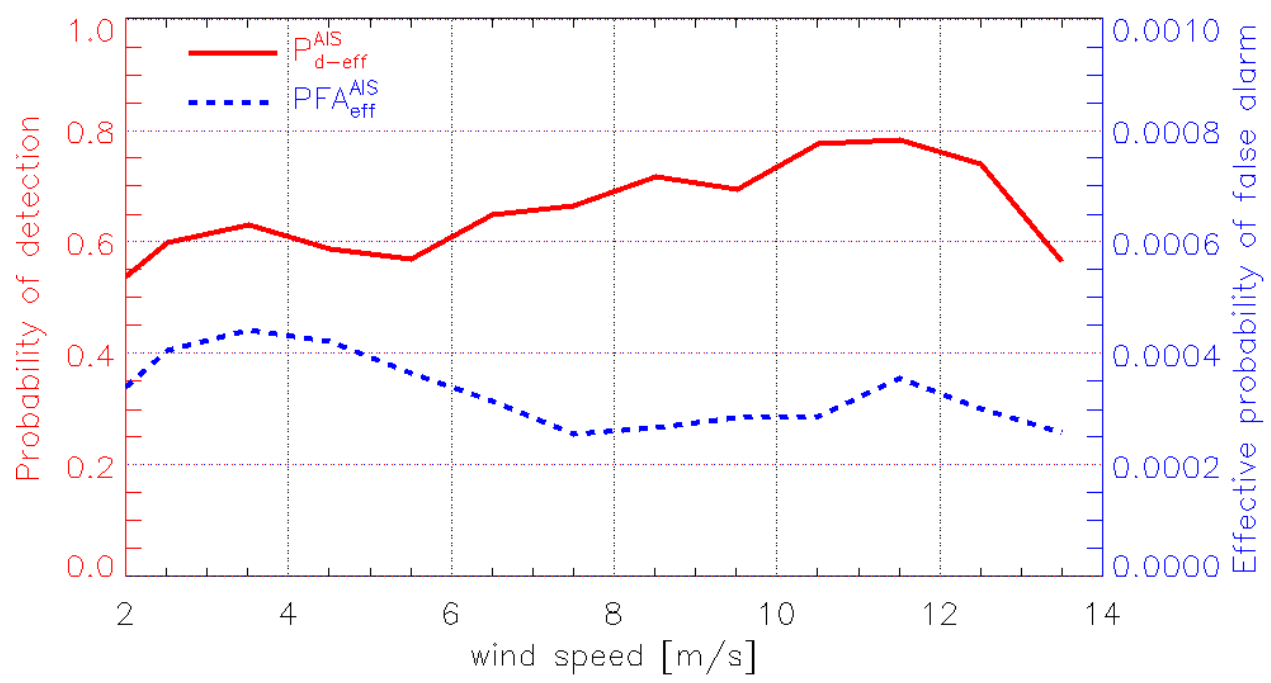

(c) 35 CSK ScanSAR Huge Region images

Figure 7: Detection rate (Gaussian detector - PFA $=10^{-7}$ ) depending on the wind speed. 


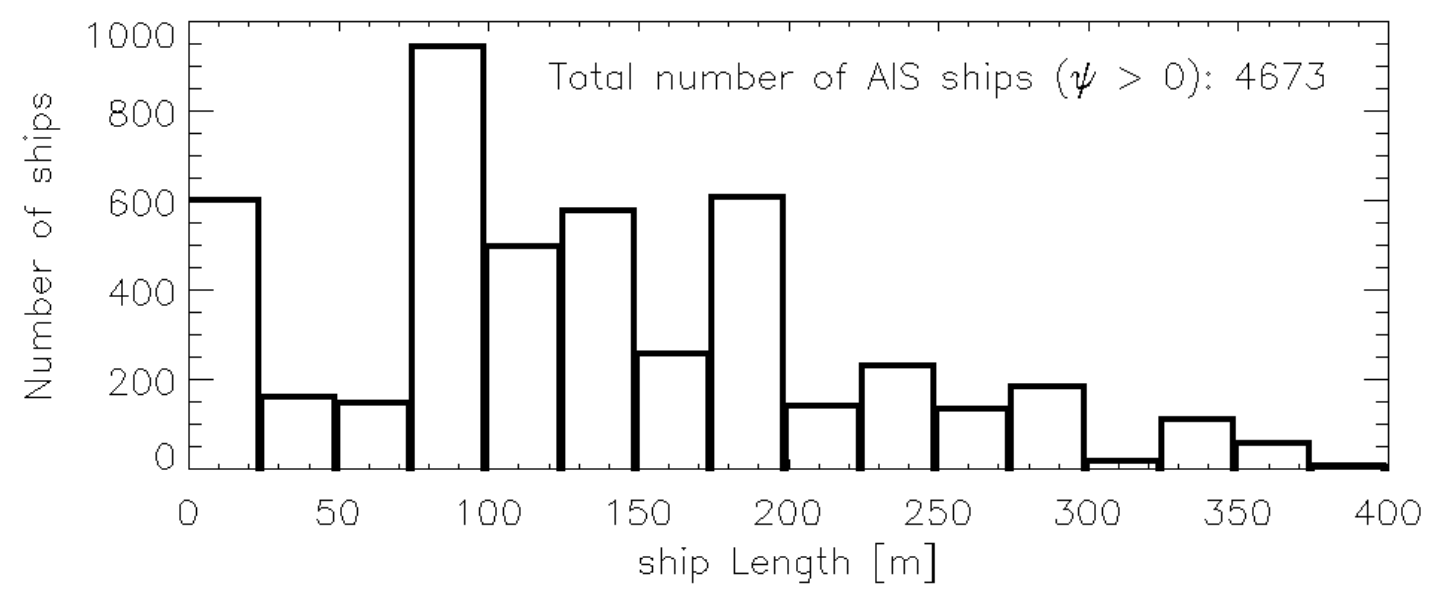

(a) $200 \mathrm{RS}-2$ ScanSAR Wide images

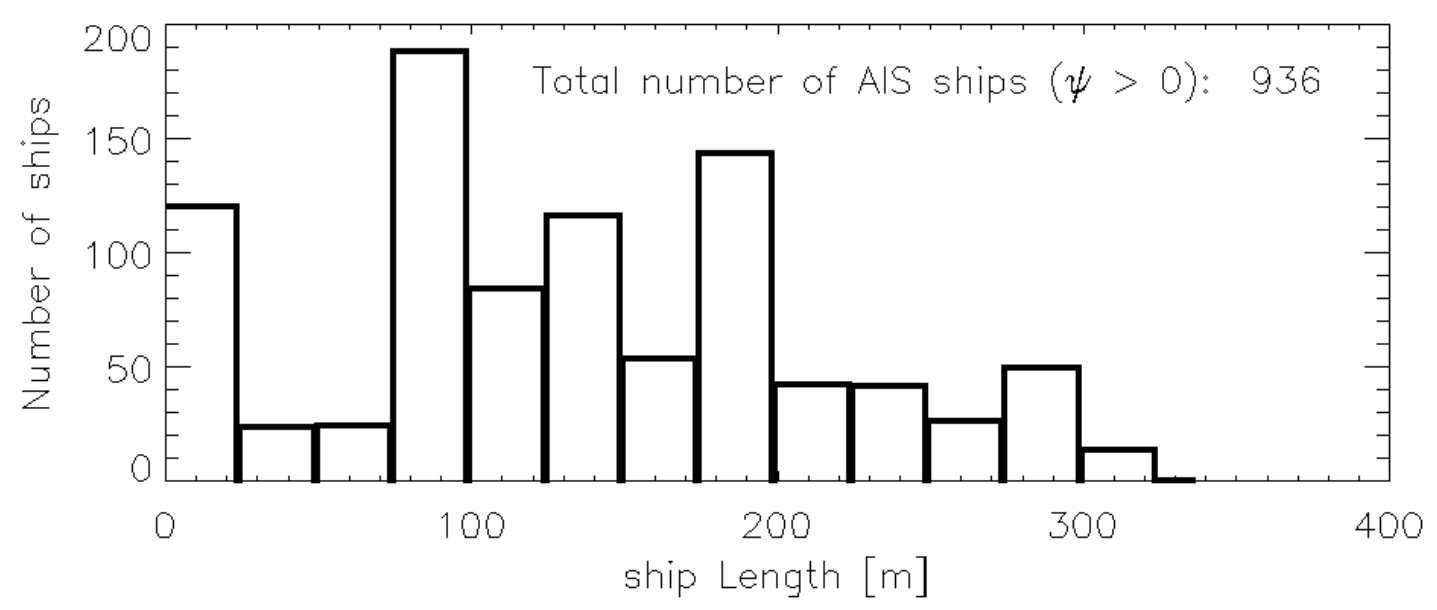

(b) 40 RS-2 ScanSAR Narrow images

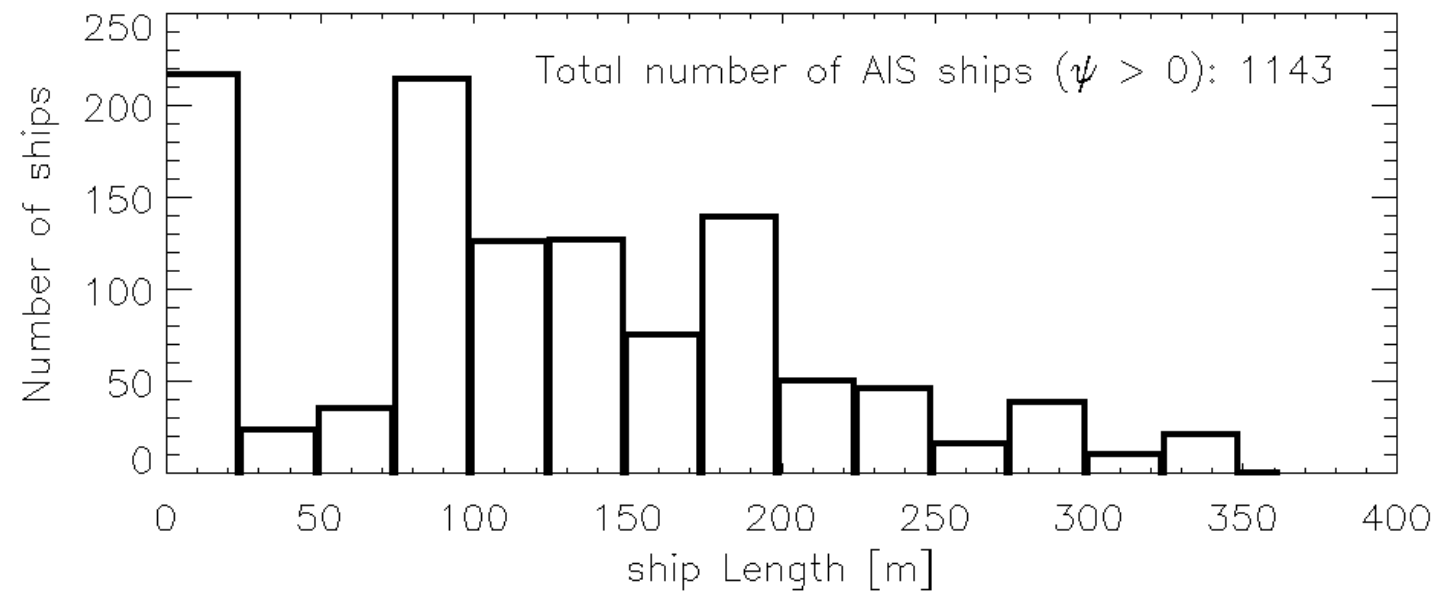

(c) 35 CSK ScanSAR Huge Region images

Figure 8: Ship length distribution over our datasets (Histogram binsize $=25 \mathrm{~m}$ ). 


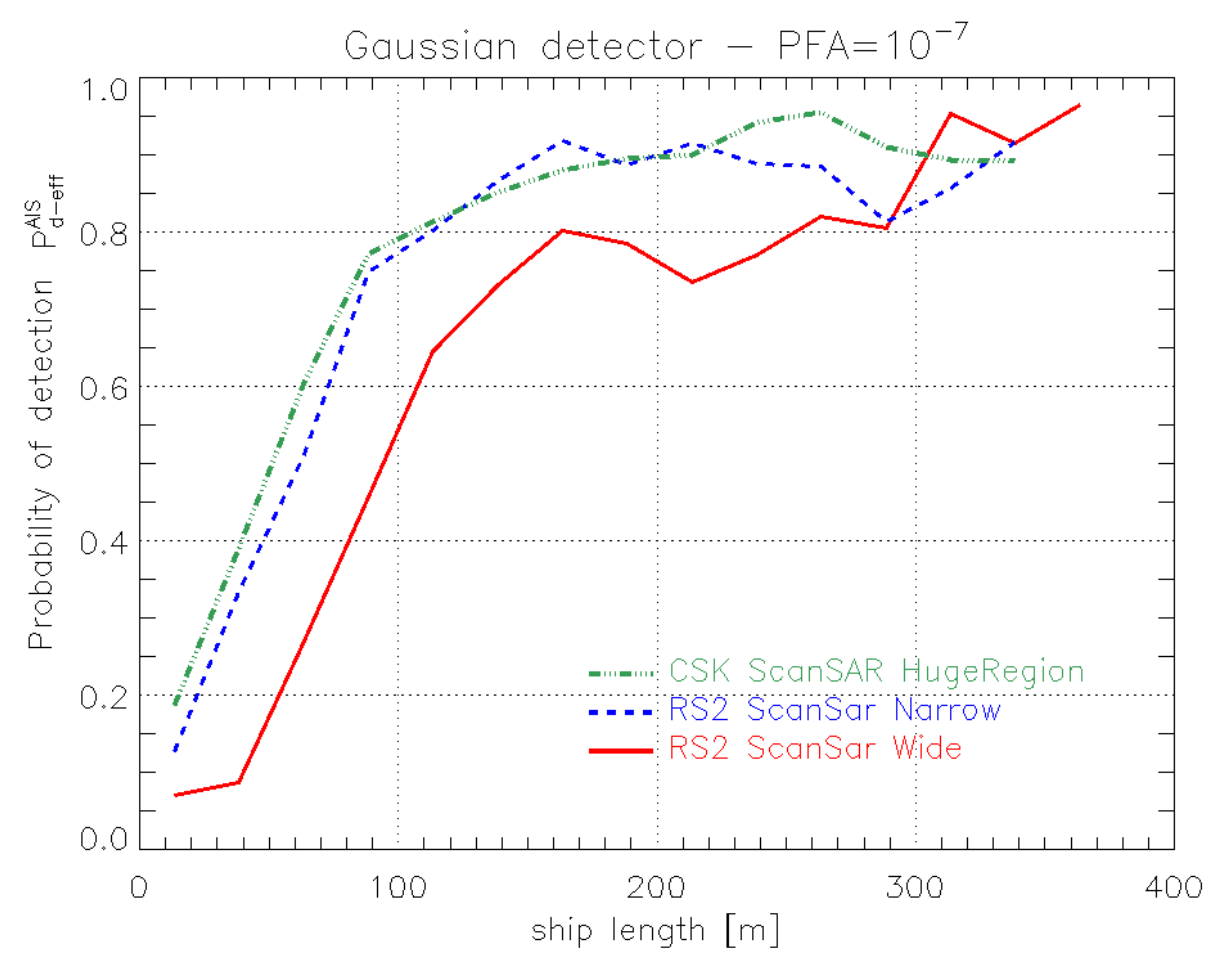

Figure 9: Detection rate (Gaussian detector - PFA $10^{-7}$ ) depending on the ship length.

for higher ship lengths $(>150 \mathrm{~m}) \mathrm{P}_{\mathrm{d} \text {-eff }}^{\text {AIS }}$ varies from 0.7 to 1 . For example, about $20 \%$ of the ships existing in the dataset, have lengths lower than $50 \mathrm{~m}$ with a detection rate tending to 0 , while ships with lengths greater than $300 \mathrm{~m}$ with a detection rate tending to 1 , represent less than $5 \%$. For the CSK dataset, the spatial resolution of SAR images $(100 \times 100 \mathrm{~m})$ is similar with the one of the RS-2 ScanSAR Wide dataset. Still, an overall comparison of the variation of $\mathrm{P}_{\mathrm{d} \text {-eff }}^{\text {AIS }}$ with ship length, shows that the CSK dataset is more advantageous for detection. For small ships (length $<50 \mathrm{~m}$ ), the detection rate varies ascending from

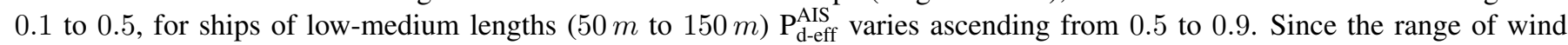
speed and incidence angle over the RS-2 ScanSAR Wide and CSK ScanSAR Huge Region datasets are relatively similar, we can state that the CSK dataset is intrinsically better than RS-2 ScanSAR Wide for ship detection.

The RS-2 ScanSAR Narrow dataset, with greater spatial resolution $(37.7-79.9 \times 60 \mathrm{~m})$ than ScanSAR Wide dataset, should provide a better detection. For instance, for ships of low-medium length $(50 \mathrm{~m}$ to $150 \mathrm{~m})$, Figure 9 shows that $\mathrm{P}_{\mathrm{d} \text {-eff }}^{\text {IS }}$ values increase from 0.4 to 0.9 , being greater than for the ScanSAR Wide dataset. Regarding small ships (length $<50 \mathrm{~m}$ ), the detection rate varies ascending from 0.1 to 0.4 , while for large length ships (length $>150 \mathrm{~m}$ ) $\mathrm{P}_{\mathrm{d} \text {-eff }}^{\text {AIS }}$ is around 0.9 . As mentioned in the previous sections, detections on RS-2 ScanSAR Narrow images is affected by the range of the incidence angle values ( to a lesser extend of the wind speed range), explaining the relatively low performances.

At this stage, it should be mentioned that the best achieved $\mathrm{P}_{\mathrm{d} \text {-eff }}^{\text {AIS }}$ are about $0.9-0.95$ for ships longer than $150 \mathrm{~m}$. Even with the worst conditions (low incidence angle and large wind speed), we would expect to achieve $100 \%$ detection for large vessels over $250 \mathrm{~m}$. The proposed methodology which automatically integrates AIS data flows to assess the detection performances could be further improved. For instance, the determined area covered by AIS stations could be affected by errors in our modelling (weather condition, temporary unavailability of AIS receivers, temporary very high density traffic). Also, matching SAR targets with AIS positions in high traffic areas could be erroneous and may require advanced interpolation/extrapolation functionalities.

\section{CONCLUSION}

In this study we have tested the performances of three ship detection algorithms. Depending on the datasets used for testing, the detection algorithms offer different performances. The systematic method used for counting good detections and false alarms in order to determine the detection rate, allows us to perform an appropriate and consistent comparison of those three detectors. The datasets used in this study contain a large number of SAR images with thousands of corresponding AIS vessels positions.

The non-parametric method, which has been applied for the first time for ship detection, gives quite satisfactory results on the lower resolution datasets (RS-2 ScanSAR Wide and CSK ScanSAR Huge Region). The Gaussian and Gamma CFAR algorithms give similar results over all the datasets, with a slightly better performance of the Gamma detector on the RS-2 ScanSAR Narrow data. 
The one-by-one scene and sensors characteristics comparisons show that the RS-2 ScanSAR Narrow dataset yields the best detection capabilities, the global detection rates over this dataset (see Fig. 3) being impacted by the incidence angle and wind speed ranges. Good detections capabilities were obtained with the CSK ScanSAR Huge Region dataset. Even though the sensor characteristics and meteorological conditions are relatively similar to the ones of the RS-2 ScanSAR Wide dataset, it induces lower detection performances, allowing to conclude that CSK ScanSAR Huge Region is better suited for ship detection at this resolution. Analyzing the detection capabilities with respect to the used dataset is valuable for operational applications, in order to chose the most appropriate type of data for different applications.

The future launch the European Sentinel-1 satellite will provide similar data being equipped with a medium resolution SAR sensor which will be used for systematic monitoring of European Waters. Two requisites for an efficient maritime surveillance with Sentinel-1 are the automatic validation of SAR-based ship detection products and an advanced methodology for sub-pixel ship characterization (vessel length).

The integration of worldwide Satellite AIS data flows should complete the ground truth data with much wider metocean conditions and then should facilitate the automatic validation. Future work also involves verifying each AIS target information with a database containing accurate ship details.

The use of the classification step reduces the false alarm rate and should provide a better ship characterization in a complete ship detection system for maritime surveillance.

\section{ACKNOWLEDGMENT}

This study is partially supported by the European Commission under Framework Programme 7 (FP7) DOLPHIN \#263079 project, the ANRT (Association Nationale pour la Recherche Technique) through a CIFRE (Conventions Industrielles de Formation par la REcherche) scholarship and by a support from the European Union, the Brittany Region and the Brest Metropole Oceane to the VIGISAT project.

\section{REFERENCES}

[1] D. J. Crisp, "The state-of-the-art in ship detection in synthetic aperture radar imagery," Intelligence, Surveillance and Reconnaissance Division Information Sciences Laboratory, Edinburgh, South Australia, Australia, Tech. Rep., May 2004.

[2] P.W. Vachon, S.J. Thomas, J. Cranton, H.R. Edel, and M.D. Henschel, "Validation of ship detection by the RADARSAT synthetic aperture radar and the ocean monitoring workstation," Canadian Journal of Remote Sensing, vol. 26, no. 3, pp. 200-212, 2000.

[3] K. Eldhuset, "An automatic ship and ship wake detection system for spaceborne SAR images in coastal regions," TGRS, vol. 34, no. 4, pp. 1010-1019, Jul. 1996.

[4] K.S. Friedman, C. Wackerman, W.G. Pichel, F. Funk, P. Clemente-Colon, and X. Li, "Validation of a CFAR vessel detection algorithm using known vessel locations," in IGARSS, vol. 4, Jul. 2001, pp. $1804-1806$.

[5] M. Cusano, J. Lichtenegger, P. Lombardo, A. Petrocchi, and D. Zanovello, "A real time operational scheme for ship traffic monitoring using quick look ERS SAR images," in IGARSS, vol. 7, Honolulu, HI, 2000, pp. 2918 - 2920.

[6] A. M. Atto, D. Pastor, and G. Mercier, "Detection thresholds for non-parametric estimation," Signal, Image and Video Processing Springer, vol. 2, no. 3, pp. 207-223, 2008 .

[7] K. El-Darymli, P. McGuire, D. Power, and C. Moloney, "Target detection in synthetic aperture radar imagery: a state-of-the-art survey," Journal of Applied Remote Sensing - SPIE, vol. 7, no. 1, 2013.

[8] K. Krishnamoorthy, Handbook of Statistical Distributions with Applications. Chapman and Hall/CRC, Jun. 2006.

[9] C. Oliver and S. Quegan, Understanding Synthetic Aperture Radar Images. SciTech Publishing, 2004.

[10] E. Magraner, N. Bertaux, and P. Refregier, "Detection in gamma-distributed nonhomogeneous backgrounds," IEEE Transactions on Aerospace and Electronic Systems, vol. 46, no. 3, pp. 1127-1139, Jul. 2010.

[11] David L. Donoho, "De-noising by soft-thresholding," IEEE TRANSACTIONS ON INFORMATION THEORY, vol. 41, no. 3, pp. 613-627, May 1995.

[12] E.H. Peterson, R.E. Zee, and G. Fotopoulos, "Wavelet-based despeckling for onboard image processing in a small satellite SAR maritime surveillance constellation," in IGARSS, Munich, Jul. 2012, pp. 1809 - 1812.

[13] N. Longépé, G. Hajduch, R. Pelich, J. Habonneau, and J.Y. Lebras, "SAR-based ship monitoring: advanced methodologies with medium resolution images (from WSM ASAR to EWS/IWS s1 mission)," in ESA - Living Planet Symposium, Edinburgh, Sep. 2013.

[14] MacDonald, Dettwiler and Associates Ltd., "RADARSAT-2 product description,” Oct. 2013. [Online]. Available: http://gs.mdacorporation.com/includes/ documents/52-1238_RS2_Product_Description_Iss1-10.pdf

[15] Italian Space Agency COSMO-SkyMed Mission, "COSMO-SkyMed SAR products handbook," Apr. 2007. [Online]. Available: http: //www.cosmo-skymed.it/docs/ASI-CSM-ENG-RS-092-A-CSKSARProductsHandbook.pdf

[16] P.W. Vachon, J.W.M. Campbell, C.J. Bjerkelund, F.W. Dobson, and M.T. Rey, "Ship detection by the RADARSAT SAR: validation of detection model predictions," Canadian Journal of Remote Sensing, vol. 23, no. 1, pp. 48-59, 1997.

[17] R. Touzi, F. Charbonneau, R. K. Hawkins, and P. W. Vachon, "Ship detection and characterization using polarimetric SAR," Canadian Journal of Remote Sensing, vol. 30, no. 3, p. 552-559, 2004

[18] G.R. Valenzuela, "Theories for the interactions of electromagnetic and oceanic waves - a review," Boundary-Layer Met., no. $13,1978$.

[19] A. Skøelv, "ERS detection of soft and hard targets at sea: what can be operationalised ?" ERS Applications, Proceedings of the Second International Workshop, Dec. 1995. 